\title{
The Public Knowledge Project: Reflections and Directions After Its First Two Decades
}

\author{
Juan Pablo Alperin, John Willinsky, Brian Owen, James MacGregor, Alec \\ Smecher and Kevin Stranack
}

\section{Introduction}

1 Getting unvarnished feedback from the community, through a third party, has given us the opportunity to see ourselves through the lens of others. This view has enabled us to reflect on our values, our activities, and our operations. We are certain about several things: At our heart is our mission to provide free open source software to anyone, anywhere, as a means of lowering barriers to creating, presenting, and sharing scholarly content. We are an academic-led initiative, and are firmly a part of the community we serve. We will never be acquired by a commercial entity. In order to continue to provide best-in-class service to our thousands of users worldwide, our not-for-profit, academicled initiative needs to operate in a way that makes best use of business strategy to ensure that our products and services are competitive with offerings of all types, and that our internal operations are efficient and productive. Our community of users is everything to us, and we will find more ways to actively support, educate, and engage them in the years ahead.

2 At the same time, it has become clear that we need to find new ways to better support our work. To this end, we present a summary of our interpretation of the findings, both the positive and negative, along with the actions we intend to take to improve how we operate. This is surely not the only way to interpret the findings report, but it reflects what we, the PKP Team, have learned about ourselves, and the vision that we have for the organization. We welcome additional input, both in response to this document and to the report that gave rise to it, as we move forward on implementing. 


\section{Our Three Pillars}

3 Through this process, a picture emerged of three areas of strength for PKP. These three distinct but interrelated activities are at the heart of PKP. Collectively, they define PKP and they explain how PKP serves its larger goal of making knowledge public:

4 1. Our open source software mission drives everything we do. We remain committed to developing excellent software and to making it freely available to all. While OJS is our flagship software, we will continue to be involved in other areas of scholarly communications, like our monograph software (OMP) and our Typesetting stack (OTS). Being an open source project is important to us because: It builds capacity around the world by encouraging a "do-it-yourself" approach; it allows PKP to benefit from outside contributions, such as translations and code; it supports more diverse use-cases, allowing users to customize their installations to suit their needs; and it demonstrates that openness is at the core of PKP.

5 2. Our research, education and advocacy are a core part of who we are. We are deeply embedded in the academic, library, and technical communities that make up higher education, and seek to both build on and contribute to our collective understanding of the ways in which that knowledge is created, circulated, and used, under the belief that the public has a right to access to knowledge. To this end, we research a broad range of issues around scholarly communications, innovate and experiment with new technologies, and work to educate and be actively involved with the academic and scholarly communication communities. Doing so helps us achieve our goals because:

a. the research builds a better understanding of the issues around scholarly communication;

b. the research and our active involvement at events keeps the PKP team embedded within the academic and scholarly communication communities we seek to affect;

c. our training materials and workshops help raise awareness and improve the quality of scholarly publishing;

d. the experimentation and innovation with technologies improves the software; and

e. being a voice in the community helps directly influence decision making and discussions.

6 3. Our PKP Publishing Services (PKP|PS) are a key way in which we support those in the community who prefer not to host software themselves, as well as to those seeking a community-based not-for-profit alternative for their publishing needs. Importantly, providing publishing services supports PKP because they:

a. provide revenue back to the organization to support the software, research, education, and advocacy work; and

b. gives us the constant feedback we need for our software to remain competitive with even the most polished commercial offerings.

7 After listening to the community, we have come to believe, more strongly than ever, that it is essential for PKP to continue to operate in all three areas. However, it has also become evident that each undertaking of PKP can no longer be done in isolation from the rest. PKP is committed to bringing these activities together in ways that reinforce each other and are philosophically coherent and consistent with the principles that underlie the open access community. 
8 Our core takeaway from this report is that PKP should strengthen the ties among its activities, and actively work to communicate more about those linkages to the community. Doing so will not only help others to assess the value of PKP, it will also provide a stronger technological, social, and financial foundation that will enable PKP to continue to have an impact that is disproportionately large for its modest budget and team size.

9 To strengthen the ties between our activities, we realize we also need to build out each area. We have taken stock of the feedback we received from the experts and stakeholders we consulted, and of our own sense of where our strengths and weaknesses are. There is much to be proud of in each realm, but also many opportunities for improvement. We summarize some of the key findings and our proposed approach to move forward below.

\section{Open Source Software}

10 The good news: OJS is the reason that a large number of journals are able to publish. Individuals and organizations around the world are able to set up an instance of OJS and operate a journal because it is free and open source, and because it has minimal server requirements. There are over 10,000 journals actively using OJS (publishing at least at least 10 articles a year, and an average of 46 articles per year). Many find OJS helpful for the ways it can be configured and the range of publications it makes possible. Those who have used or seen OJS3 are very pleased with the more modern design and upgraded features.

11 The less-good news: Some are frustrated by what they perceive to be an unnecessarily slow pace of change. Many perceive OJS as being outdated and rigid, both in its look and its workflow, based largely on their experience with OJS2. Some have stated they do not feel PKP functions as fully "open source" as it could and should be more actively seeking code contributions from others. Some feel OJS is "too heavy" and requires using or dealing with more workflow elements than are needed. There is little awareness and lower levels of adoption of the other PKP software (e.g., OMP and OCS).

12 Next steps: We plan to actively encourage open source contributions from the community. Doing so will increase the speed at which we can deliver new features and respond to the needs of the community, like we have seen with OJS being translated by volunteers into dozens of languages. To do this, we will:

a. Find ways to call attention to the many contributions our community continues to make to our code and to our documentation;

b. Review and improve documentation for the developer community;

c. Revise and redesign the online spaces (such as the PKP Forum and Github), so that it can serve as an obvious way to encourage recommendations for new features, have the community vote on them, and track progress as new efforts progress;

d. Specify discrete and actionable work "packages" or assignments for outside contributors;

e. Dedicate PKP developer time to facilitate code contributions from those that express interest in customizing their own installations;

f. Host more developer-centric events (e.g., code sprints);

g. Decrease the code complexity by incorporating third party software and updating/ streamlining code to be more transparent. 
13 We also plan to make our software easier to use and adopt. We are certain that OJS delivers value (10,000 journals cannot be wrong!). But still, people have issues with how it looks, operates, and what it can do. We will address these issues by:

a. Develop tools, documentation, and service packages to make it easier for existing OJS2 users to migrate to OJS3, so they can enjoy its full benefits;

b. Review the OJS interfaces and workflow to see if there are ways of offering a more streamlined experience for those not requiring all the functionality it offers;

c. Communicate more effectively the modular and customizable aspects of OJS 3, by offering visuals, documentation, and other guidance on ways it can be used in part or in whole;

d. Invite more community input at the vision, planning, and priorities stages;

e. Make it easier for users to see how OJS can function in harmony with other popular software and infrastructure, including open source repositories (e.g., DSpace, Islandora, Samvera) and centralized services (e.g., Crossref, ORCID).

\section{Research, Education, and Advocacy}

14 The good news: There is a high degree of trust, admiration and respect for PKP. The vast majority of those we consulted held PKP in high regard, and were appreciative of the work that we do. We are actively publishing and presenting at academic venues around the world, with both invited and peer-reviewed activities; our research is widely read and used to inform the collective understanding of scholarly communication (e.g., Alperin and Rozemblum, 2017; Alperin, Gomez, and Haustein, in press; Willinsky, 2017; Willinsky and Alperin, 2018). We are a strong voice for scholarly publishing in the academic community, with PKP team members serving on editorial boards and advisory groups of important initiatives such as SPARC, Crossref, Open Library of Humanities, HIRMEOS, ScholarlyHub, DOAJ, Redalyc, and the Library Publishing Coalition. Our educational offerings have made a significant contribution towards professionalizing the work of new editors and emerging publisher. We have successfully moved some experimental services, like the PKP Index and the PKP Preservation Network into production offerings, open to all journals that use OJS. We are developing new community-based publishing models, whether nationally with Coalition Publi.ca in Canada or within disciplines, such as Libraria in anthropology and working with similar initiatives around the world, e.g. Journal.fi (Finland).

15 The less-good news: There is a very limited awareness of PKP's activities outside of our developing OJS. Some believe that PKP's software needs to be more innovative and keep up with the times. Some have expressed frustration with their efforts to collaborate with PKP, following promising conversations with PKP team members at events and meetings. Our capacity to deeply interact with a very large and geographically diverse community has been limited to attending and putting on conferences and to the PKP Forum. PKP's educational content requires regular updating and expansion to better serve the community, and more effort is needed to raise awareness of activities in this area. 
Prototypes, like article-level metrics and automated XML typesetting, have not yet made it into production, despite their importance to the community.

Next steps: PKP's strong reputation gives us the opportunity to play a leadership role in supporting the academic community, but to do so, we need to:

a. Reinvest in community outreach, as a formal activity, offering more ways for us to stay in touch with our users, more ways to support them, and help them to help each other;

b. Re-evaluate our educational activities to foster a shared understanding of the values and practices between PKP and the community;

c. Overhaul our web presence, documentation and other materials to be more inviting of input and dialogue with the community, as well as to better showcase the work and activities PKP is already involved with;

d. Expand the reach and diversity of PKP's community-based committees with a particular emphasis on guiding PKP's work across all areas;

e. Build on the global reach of PKP by hosting or helping to organize events with user groups around the world;

f. Explore feasible and reliable strategies for OJS journals and their users to avoid "predatory journal" designations by introducing public-facing verification systems, including thirdparty checks, metrics, and AI to establish editor, editorial board, reviewer and review integrity.

We also need to formalize processes for research and innovation activities to improve our offerings. We have been successful as a research group, but our research does not always make its way into our offerings. Similarly, we have had mixed success in moving experimental services into production. To strengthen the ties between our research, software, and services, we will:

a. Develop and seek funding for a research agenda that address pressing needs of the academic community, while simultaneously providing useful intelligence to further PKP's development;

b. Create something akin to a "PKP Labs," as a means of identifying, building and testing innovative projects, supported by internal and external funds.

\section{PKP Publishing Services}

The good news: We have created a publishing services offering (PKP|PS) that has continued to grow since it was started in 2007 , to a point where today it supports approximately 50 percent of PKP's annual operating expense. Our clients report a high level of satisfaction with the degree to which we are able to customize our software to suit their needs. We serve a diverse set of clients, including everything from small, independent publishers with one journal, to major society publishers with many high-profile journals.

The less-good news: There is limited awareness of PKP|PS and the services it offers. There are many competitive services available that host OJS; some are in the academic space, some are commercial offerings. Most do not contribute anything back to PKP. We know the degree of support top-tier publishers require, and to expand our offering, we will need to substantially increase our staff support.

Next steps: Our publishing services have grown to make up over half of our operating revenues, and they have done so without any targeted promotion. Although some clients 
seem to find us through word of mouth or through our webpage, too many potential clients are unaware of our services. We will promote and grow our services by:

a. Having members of the PKP|PS team participate in industry conferences, as well as academic events, for the purposes of promotion, hiring new staff if necessary;

b. Developing and executing a full marketing strategy that is consistent with PKP's mission and values, while simultaneously extolling the quality of PKP|PS.

21 Our current clients include single-journal publishers and multi-journal publishers, commercial and non-profit organizations. However, we are aware that certain types of publishers do not see us as an obvious choice, so we have decided to:

a. Developing service packages that can be customized to address the needs of a certain type of user/potential client;

b. Build partnerships with other service providers, or expand our own services, to offer additional options to all clients, including professional publishing and editorial support services, theming, and customization;

c. Present an array of service options and packages in ways that make it easier for the wide range of people who make purchase decisions.

The publishing services team already runs a successful operation that generates surplus revenue, but more can be done to improve its efficiency and increase the surplus that supports other PKP activities. We will improve operations by:

a. Investing in building tools that the PKP|PS team can use to further automate installing, upgrading, and monitoring journals;

b. Offer an option to use a shared installation (i.e., cloud-based) for journals that do not require customizations to minimize the maintenance overhead;

c. Revisit our pricing model, to make it more appealing for large institutions and publishers to host a large number of journals with us.

\section{A Way Ahead}

23 After two decades of being an academic-led "project" within the university context, the PKP organization has successfully matured into a reliable, secure, and evolving organization with a range of related goals and activities, and a globally recognized contribution to make to scholarly communication and public knowledge. We have formed a dedicated team, many members of which have now been with PKP for well over a decade, and all of whom are experts in our software and in supporting our community around the world in various ways. That said, our internal operations are ready for a new phase of growth and structure. In order for the plans outlined in this report to take flight, we understand we will need to make internal changes by, for example, supplementing existing staff with those who can bring additional skills to our team.

We are also committed to greater coordination among open infrastructure projects supporting all phases of scholarly communication, not only around interoperability among these systems, but around ensuring that there are open, community support and tracking systems at each step of the research life cycle. Through such means, and in cooperation with libraries and funders (see Willinsky and Rusk, in press, for one financial model), we see the means of achieving a sustainable and universal open access that operates in conjunction with open data, open educational resources, and open infrastructure (based on open source software). 


\section{Organizational Structure and Governance}

Our organization currently includes an internal management team and a few advisory groups. We are based at Simon Fraser University, in Canada, with roots in both SFU Library and the School of Publishing. We plan to keep these elements in place going forward, but are committed to revisiting the governance structure, the makeup and mandate of the advisory groups, and the mechanisms we use to gather ongoing feedback and direction from our community.

Internally, we have already begun to see how thinking about PKP's work around the three pillars outlined above can be reflected in the financial management of the organization. As we seek to strengthen each pillar of activity, we are ensuring that each area operates under its own internal logic, with specific goals that can be tracked. By having these be reflected in the financial management, we are ensuring each area is adequately resourced, and is not made to suffer at the expense of the others. Conversely, we are also making explicit the direct and indirect supports that each provides the others, so that when resources need to shift from one priority to another, it is done so with a clear rationale and well-defined parameters.

Externally, we recognize the need to restructure our decision making to have greater involvement from the community. We see potential in a membership model, with members having voting rights to help us determine key elements on our development roadmap and the project's overall direction. The actions outlined above are also largely oriented to capturing more community feedback, regardless of membership, and so we intend to formally bring that feedback into decision-making going forward.

\section{Internal and external communication}

One of the main findings of this study was that that we need to better communicate how PKP contributes to open scholarly publishing. Communicating who we are and what we do is built into the proposed actions of each of the pillars above, but we feel it is important to highlight separately, as it is essential that PKP also present the unified picture of how the pieces fit together. To do so we need to develop a formal communication strategy that can be used within the organization to ensure we have a shared understanding of our goals and activities, and externally so that the scholarly publishing and academic communities can fully benefit from and appreciate the work that we do.

This communication strategy needs to be bidirectional, and will require one or more additional team members who can actively do outreach at major community events, participate in important meetings, and regularly solicit frank feedback from stakeholder groups on directions and details (like this project did).

\section{Sources of support}

PKP has just concluded a five-year sustainability strategy that saw our operating budget grow substantially, and which has put us on much firming footing going forward. This growth has come in large part from the PKP's Publishing Services (PKP|PS), but continues 
to be supplemented by our sustainers program and by significant grant funding. We have also benefited from community contributions through things like translation of our software and some code contributions. However, we are convinced that we are not maximizing the potential in any of these areas.

The actions outlined above will go a long way to increase the in-kind support we get through community contributions. Our inability to grow the pool of contributors substantially over the years has limited our growth, and we are certain that by having dedicated personnel who will be in charge of outreach, documentation, and development (in the specific ways outlined above), we will translate into growth in the contributions we receive in various forms (e.g., translations, code, hosting PKP events, etc.). The value of these contributions, we expect, will far outweigh the efforts and costs necessary to generate them.

However, to grow the team with people who are capable of encouraging and supporting more community contributions, we need to have the necessary financial resources. We see potential in both models recommended: a membership model that can bring both financial and in-kind contributions (as well as feedback and direction, as noted above), and through the growth of PKP|PS.

To grow our membership model, we are reviewing ways to make participation more attractive to a wide number of our users. Some may want to affiliate with PKP for benefits like discounted registration for PKP conferences and workshops. Others-those with deeper ties to PKP-may want to support the organization financially. We will review our key relationships, and seek to establish closer ties to organizations that are built upon our software, to see how their success can also help fuel our ongoing support of their work. This work will itself require dedicated people to build relationships, but after an initial investment, the financial contributions of members should make this position selfsustaining.

To grow our PKP Publishing Services, we need to first ensure that people become aware of the services we offer, and subsequently review our offerings to provide the level of support that even the largest and most sophisticated publishers expect. We have been fortunate until now to not have to actively promote our services, but we realize that this has limited our capacity to grow. Growing PKP|PS will require bringing on, at the very least, a dedicated business development manager and customer service support specialists. The business development manager will be seeking clients from organizations that support multiple journals, and will actively work with university-based publishers to introduce them to our offerings, as well as seeking out other segments for growth. Like with the personnel necessary to grow the membership model, the costs associated with these positions should be self-sustaining through growth in the business after an initial investment. Moreover, the outreach that helps grow the business will also be a key element in ensuring that those who use OJS and our other software feel fully a part of the broader PKP community.

Being an academic-led project, we will also continue to pursue grants as a way to support the research and innovation of PKP, but hope to use the new organizational structure to remove the reliance on such grants for core operations. Grants, unless specifically intended for operations, should be focused on the research activities of PKP, with the intended benefit to other areas, but without becoming a source of direct support for nonresearch activities. Taken together, these efforts to both raise revenues, streamline 
processes, and expand the membership base of the organization will permit the PKP to quickly expand the speed of development, and to remain in close touch with the very people it was created to serve.

\section{Conclusion}

This study served as an inspirational moment for our team in its commitment to supporting greater access to research and scholarship into a third decade. It has helped us gain insight into what distinguishes PKP's contribution and what people value about our approach. We are more certain than ever that PKP should sustain its efforts in the three designated areas, while realizing that there is always more to do in fulfilling the resulting responsibilities:

a. to communicate the range and complexity of our undertakings;

b. to be responsive and attentive to academic, scholarly publishing and open sources communities; and

c. to collaborate with a growing number of organizations to advance the quality and openness of research and scholarship.

After a thorough consultation with members of the scholarly publishing community, Nancy Maron concluded that PKP has strengths to build on in undertaking the change that is needed to better serve its community:

PKP has developed a deep and loyal global following over its nearly twenty years of operation. It is poised today to squarely addresses its challenges and leverage its strengths into a dynamic plan for the future. The next steps will almost certainly involve a good deal of change, and this may not be easy. But publishers of all types in countries around the globe, continue to seek ways to create, consume, publish and share information, and the Public Knowledge Project, through its mission, its software, and the support of its community, is in a position to help them as needs continue to evolve (Maron, 2018).

Our plan is to use what we have learned from the Community Consultation that Nancy Maron conducted to not only catch up with the growth in the global use of our software, in terms of our publishing services and support, but to push the thinking about ways of improving scholarly communication even further, in pursuit of such goals as universal open access and community control of scholarly publishing infrastructure.

Going forward, we intend to facilitate increased community contributions and input throughout the areas in which we work; to strive for more internal coherence and crossover among what we now think of as PKP's three pillars; to give the library community and granting agencies more reasons to sponsor both our publishing innovations and our software upgrade cycles. More specifically on this last point, we see exciting opportunities ahead for developing editorial efficiencies for improved research quality, reader enhancements through design improvements, stronger integration of infrastructure for improved scholarly communication at every phase of the research process. We can also see now that our aim is to build a publishing service that offers both a level of professionalism (in its responsive service focus) and a commitment to being of, as well as for, the academic community of which it remains proud to be an integral part. 
BIBLIOGRAPHY

\section{References}

Alperin, J.P., \& Rozemblum, C. (2017). “The Reinterpretation of the Visibility and Quality of New Policies to Assess Scientific Publications." Revista Interamericana de Bibliotecologia, 40(3). http:// dx.doi.org/10.17533/udea.rib.v40n3a04

Alperin, J.P., Gomez, C., \&Haustein, S. "Identifying diffusion patterns of research on social media." (In press). Public Understanding of Science.

Maron, Nancy (2018). Understanding the Audience of the Public Knowledge Project's Open Source Software: Findings from the Planning Grant from the Laura and John Arnold Foundation. Yonkers: Bluesky to Blueprint.

Willinsky, J. (2018). The Intellectual Properties of Learning: A Prehistory from Saint Jerome to John Locke. Chicago: University of Chicago Press.

Willinsky, J. and J.P. Alperin. (2018). "The academic ethics of open access to research and scholarship." In Volume 2 of B. Smart, K. Peggs, and J. Burridge (Eds.), Critical Social Research Ethics. Los Angeles: Sage.

Willinsky, J. and M. Rusk, M (In press). "If funders and libraries subscribed to open access: The case of eLife, PLOS, and BioOne." College and Research Libraries. Preprint: https://goo.gl/qg6631

\section{ABSTRACTS}

As the Public Knowledge Project (PKP) enters its third decade, it faces the responsibilities of supporting the more than 10,000 journals using its software and are dependent on PKP continuing to develop the code. In the fall of 2017, PKP, with the support of the Arnold Foundation, contracted the consulting services of Bluesky to Blueprint, with its principal Nancy Maron embarking on an exploration of PKP's standing and prospects among a sample of those involved in scholarly publishing, including current, former, and potential users of its software (Maron 2018). This paper presents BlueSky's findings and PKP's responses in what may serve as a lesson on the maturing of, and challenges faced by, an open source software project seeking to sustain increased global access to research and scholarship. 
INDEX

Keywords: Scholarly publishing; Open access; Open sources software

\section{AUTHORS}

\section{JUAN PABLO ALPERIN}

Simon Fraser University, Canada

JOHN WILLINSKY

Stanford University, United States

john.willinsky@stanford.edu

https://ed.stanford.edu/faculty/willinsk

(corresponding author)

BRIAN OWEN

Simon Fraser University, Canada

JAMES MACGREGOR

Simon Fraser University, Canada

ALEC SMECHER

Simon Fraser University, Canada

\section{KEVIN STRANACK}

Simon Fraser University, Canada 